\title{
An Uncommon Presentation of a Common Illness: Epstein-Barr Virus (EBV) Infection Case Report and Review of Literature
}

\author{
Mark Yarchoan ${ }^{1}$, David Flynn ${ }^{2}$, Ingrid Hyder ${ }^{3}$, Subhasish Bose ${ }^{4}$ \\ 1. The Sidney Kimmel Comprehensive Cancer Center, Johns Hopkins 2. Department of Anesthesiology \\ and Critical Care, University of Pennsylvania, Philadelphia, PA 3. Department of Radiology, Pennsylvania \\ Hospital, Philadelphia, PA 4. Section of Nephrology and Kidney Transplantation, Department of \\ Medicine, Temple University
}

$\square$ Corresponding author: Mark Yarchoan, mark.yarchoan@jhu.edu

Disclosures can be found in Additional Information at the end of the article

\section{Abstract}

Epstein-Barr Virus (EBV) infection has a diverse spectrum of clinical presentations. We encountered a previously healthy young female adult with positive EBV Viral Capsid (VCA)-IgM serology and heterophile antibodies who presented with abdominal pain, mesenteric adenitis, and elevated liver enzymes without the more common manifestations of EBV infection. A review of the clinical and laboratory manifestations of EBV infection is provided, focusing on the association between EBV infection and elevated liver enzymes and abdominal pain.

Categories: Internal Medicine, Radiology

Keywords: abdominal pain, mesenteric adenitis, elevated liver enzymes, hepatitis, epstein-barr virus (ebv)

\section{Introduction}

When infection with the Epstein-Barr Virus (EBV) occurs during young adulthood, it can cause infectious mononucleosis (IM), which is classically marked by a triad of fever, pharyngitis, and lymphadenopathy. Although many cases of IM feature all three classic symptoms, primary EBV infection may have a diverse spectrum of clinical presentations. Likewise, the severity and duration of symptoms of EBV-associated IM may vary significantly [1].

The clinical and laboratory manifestations of EBV-associated IM have been described in a number of classic series; however, many of these studies did not include serologic evidence of EBV infection. More recently, Grotto and colleagues described the natural course of EBVassociated IM by prospectively studying 330 patients serving in the Israel Defense Force (IDF) with clinically suspected IM who were also EBV-Viral Capsid (VCA)-IgM positive. In this series, the most common manifestations of primary EBV infection included lymphadenopathy (89\%), malaise (86\%), sore throat (83\%), headache (83\%), fever (79\%), anorexia (74\%), and splenomegaly (53\%). Only $68 \%$ of patients in this series had the classic triad of fever, sore throat, and lymphadenopathy [2].

\section{Case Presentation}

A 23-year-old obese female with no significant past medical history presented to the emergency room complaining of a one day history of 10/10 abdominal pain radiating to her back, nausea, and non-bloody non-bilious vomit. The pain seemed to have no association with

How to cite this article

Yarchoan M, Flynn D, Hyder I, et al. (February 25, 2011) An Uncommon Presentation of a Common Illness: Epstein-Barr Virus (EBV) Infection Case Report and Review of Literature. Cureus 3(2): e23. DOI $10.7759 /$ cureus. 23 
food or drink, and was not relieved by ibuprofen or over-the-counter antacids. Of note, the patient had experienced a similar episode two weeks earlier, and had also presented to the emergency room at that time. Her pain during this previous admission had improved rapidly, and the patient was discharged 12 hours later. However, her laboratory data from this encounter was notable for elevated liver transaminases, with an AST of 231 and an ALT of 114.

During the present admission, the patient denied recent use of alcohol, acetaminophen, new medications, or alternative therapies. She also denied intravenous drug use, had never received a transfusion, and was up-to-date on vaccinations. Her family history was non-contributory; specifically, she denied family history of rheumatologic disorders or autoimmune hepatitis. She reported that she had not been sexually active for two months, and had recently had a menstrual period.

The patient's vital signs were normal, with a temperature of $98.6^{\circ} \mathrm{F}$. Her physical exam was notable for a soft and obese abdomen, with diffuse upper abdominal tenderness to medium palpation with some rebound tenderness. Her physical exam was otherwise normal. She had no cervical motion tenderness and bowel sounds were normal. She also had no jaundice, the liver edge and spleen were not palpable, and her pharynx was unremarkable. Laboratory values were significant for high liver transaminases and bilirubin: AST 690 (ref 15-41), ALT 438 (ref 14-54), total bilirubin 1.9 (ref 0.2-1.2), and direct bilirubin 0.7 (ref 0.0-0.2). Protein, albumin, PT, and PTT were within normal limits. Also, the patient was anemic with a hemoglobin of 10.6 $\mathrm{g} / \mathrm{dL}$ and had a white cell count of 13.9 thousand/uL.

A hepatitis work-up, including viral hepatitis (A, B, C), autoimmune hepatitis (SMA, ANA, AMA), iron studies, copper studies (ceruloplasmin), and alpha 1 antitrypsin, was unrevealing. Other laboratory studies, including a urine analysis, hemoglobin electrophoresis, beta-HCG, HIV, chlamydia, and gonorrhea, and stool culture were also normal or negative. However, her EBV mononucleosis screen was positive.

A complete pelvic ultrasound supplemented by endovaginal imaging was performed and was normal. A computed tomography (CT) scan of the abdomen and pelvis performed utilizing 5 $\mathrm{mm}$ axial and $3 \mathrm{~mm}$ coronal reconstructions following the uneventful administration of oral contrast and $125 \mathrm{cc}$ of intravenous Isovue 370 showed several prominent subcentimeter mesenteric nodes in the right lower quadrant of uncertain significance, suggestive of mesenteric adenitis (Figure 1). The liver, spleen, pancreas, and adrenal glands were unremarkable. 


\section{Cureus}

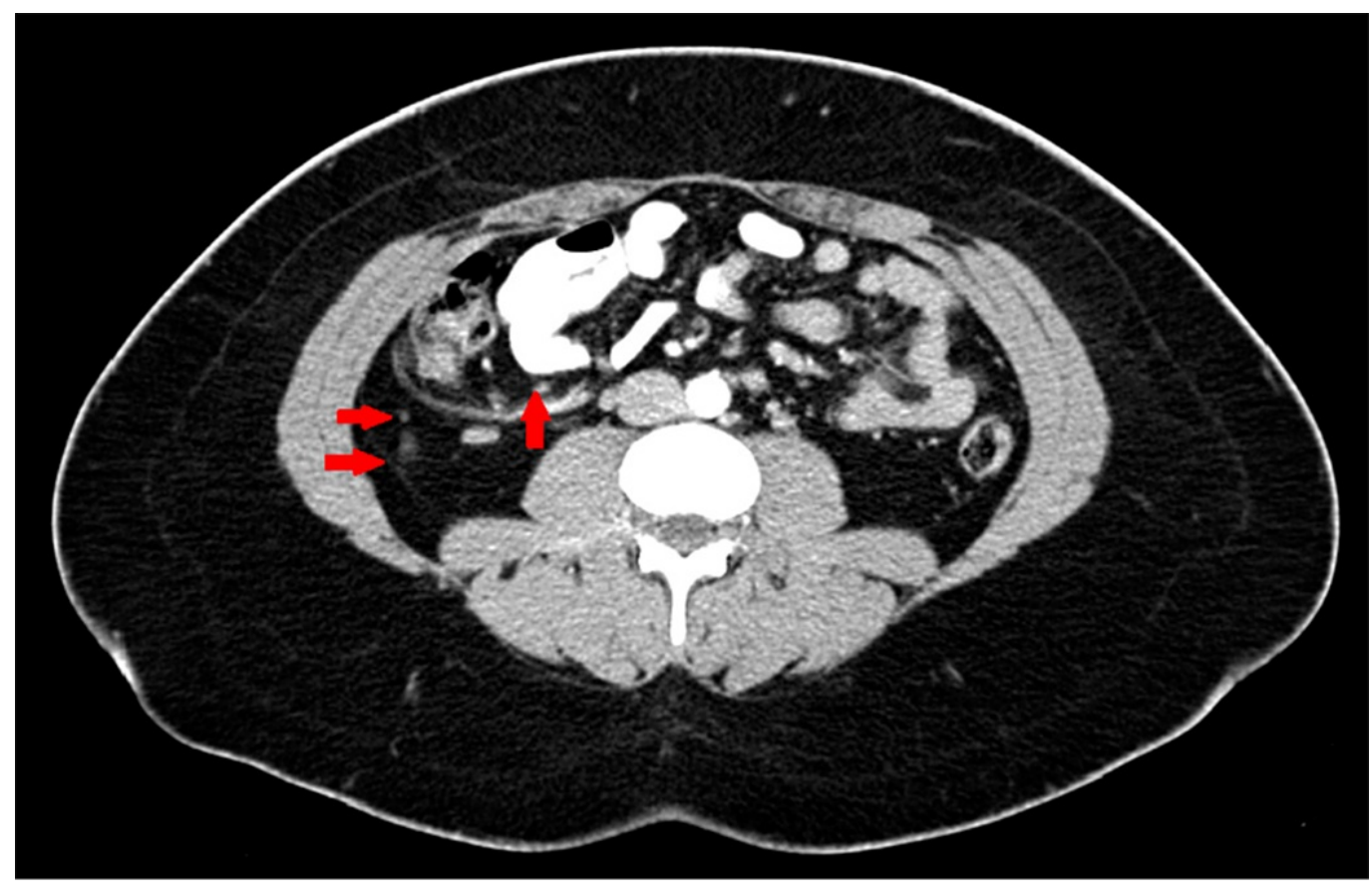

FIGURE 1: Contrast CT of the abdomen showing lymphadenopathy most apparent in the right lower quadrant.

The patient's symptoms improved significantly, and her liver transaminases returned to nearnormal by the time of discharge five days after admission (Figure 2). Interestingly, AST levels returned to baseline more rapidly than ALT levels. The patient's white blood cell count rose to 14.4 THO/uL over the first three days of admission before descending to near normal. Two weeks after admission, the patient was contacted by phone and reported complete resolution of her symptoms. She declined the opportunity for clinical follow-up, and therefore no subsequent EBV titers are available for consideration.

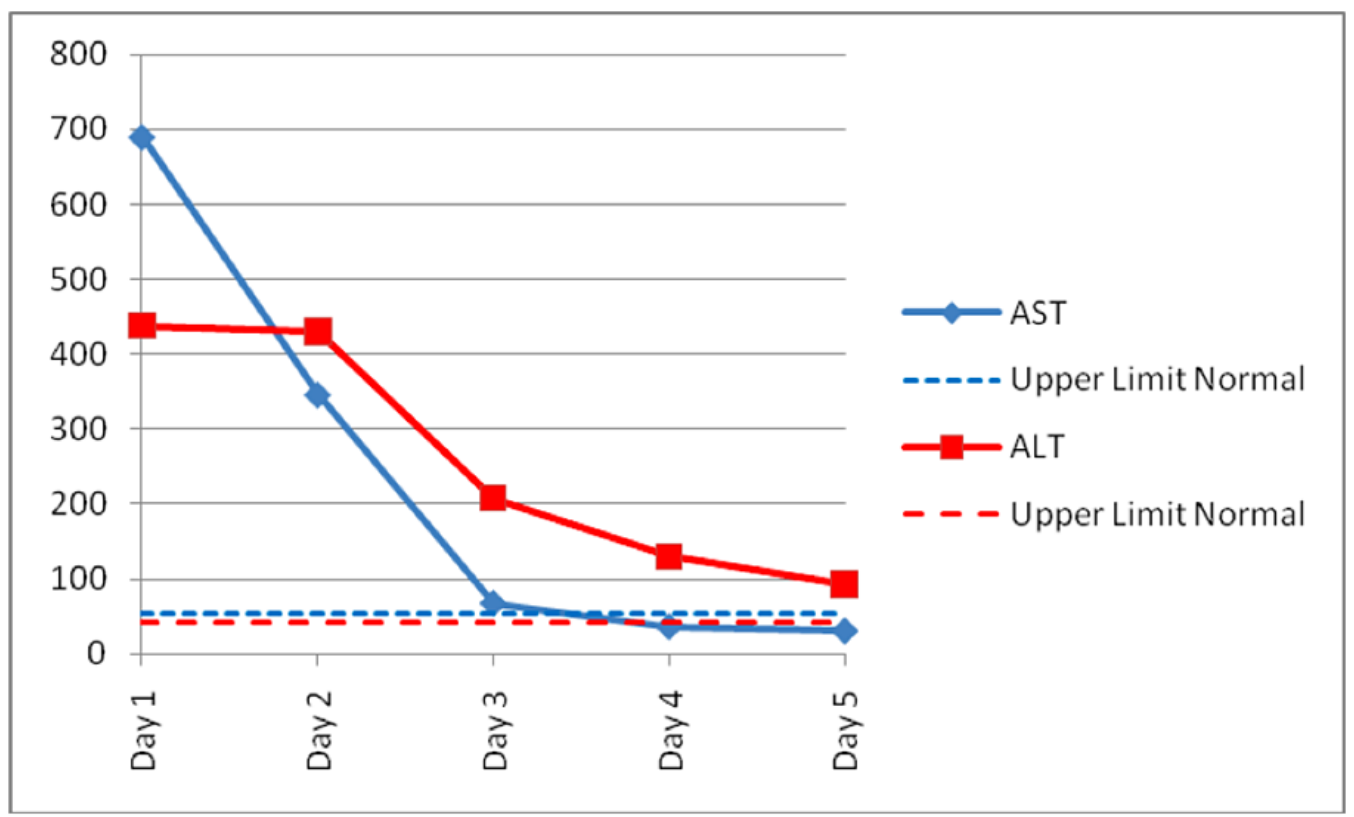

FIGURE 2: AST and ALT values during the patient's admission. 


\section{Discussion}

Hepatic involvement is a relatively common manifestation of acute EBV infection. In Grotto's series, $58 \%$ of patients had elevated liver enzymes, a number that is similar or slightly lower than in other published series [1-4]. In general, aspartate aminotransferase (AST) elevation predominates over alanine aminotransferase (ALT) elevation but rarely rises above $500 \mathrm{U} / \mathrm{L}$ in IM. Alkaline phosphatase (Alk Phos) and gamma-glutamyltransferase (GGT) are also typically elevated above $85 \mathrm{U} / \mathrm{L}$ and $25 \mathrm{U} / \mathrm{L}$, respectively, and bilirubin may be elevated as well. Liver enzymes usually reach a maximum elevation within 18 days of the onset of EBV-induced IM before returning to baseline [4]. There is some disagreement in the literature with regard to how frequently hepatomegaly or jaundice is observed. In Grotto's series, hepatomegaly was observed in more than one-third of IM patients and $16.7 \%$ were considered to be jaundiced [2], while other series have found these manifestations of IM to be uncommon or absent [5]. EBVassociated hepatitis is typically benign and self-limited, although fulminant hepatitis has been described [6], and chronic EBV infection may play a role in the development of autoimmune hepatitis [7].

Abdominal pain and mesenteric adenitis have not been widely reported in acute EBV infection. Abdominal pain in association with infectious mononucleosis may suggest splenic rupture [8]; however, the patient's spleen in the present case appeared normal on CT imaging. Although abdominal pain is not a classic symptom of EBV infection, there are reports of EBV-associated acute abdomen [9], and EBV infection may also be associated with recurrent abdominal pain. In a recent study of 518 school children with recurrent abdominal pain, 55\% had serologic evidence of acute or prior EBV infection, a number that is greater than would be expected in this population [10]. The present case report supports the theory that EBV may directly (through infection of target B cells) or indirectly (by promoting immune T-cell expansion) cause mesenteric adenitis and abdominal pain.

\section{Conclusions}

The described case of EBV-associated hepatitis, with possible mesenteric adenitis, is unusual because the patient did not present with the most common infectious mononucleosisassociated symptoms of enlarged cervical lymph nodes, sore throat, fever, malaise, or headache. In fact, her only commonly appreciated symptoms of acute EBV infection were anemia, abdominal pain, nausea, and elevated liver enzymes. The case serves as a reminder that EBV infection has a diverse spectrum of clinical presentations, and should be considered in the differential diagnosis of all young patients with elevated liver enzymes.

\section{Additional Information \\ Disclosures}

Human subjects: Consent was obtained by all participants in this study. Conflicts of interest: In compliance with the ICMJE uniform disclosure form, all authors declare the following: Payment/services info: All authors have declared that no financial support was received from any organization for the submitted work. Financial relationships: All authors have declared that they have no financial relationships at present or within the previous three years with any organizations that might have an interest in the submitted work. Other relationships: All authors have declared that there are no other relationships or activities that could appear to have influenced the submitted work.

\section{References}


1. Straus SE, Cohen JI, Tosato G, Meier J: NIH conference. Epstein-Barr virus infections: Biology, pathogenesis, and management. Ann Intern Med. 1993, 118:45-58.

2. Grotto I, Mimouni D, Huerta M, Mimouni M, Cohen D, Robin G, Pitlik S, Green MS: Clinical and laboratory presentation of EBV positive infectious mononucleosis in young adults. Epidemiol Infect. 2003, 131:683-9.

3. Finkel M, Parker GW, Fanselau HA: The hepatitis of infectious mononucleosis: Experience with 235 cases. Mil Med . 1964, 129:533-8.

4. Horwitz CA, Burke MD, Grimes P, Tombers J: Hepatic function in mononucleosis induced by Epstein-Barr virus and cytomegalovirus. Clin Chem. 1980, 26:243-6.

5. Rea TD, Russo JE, Katon W, Ashley RL, Buchwald DS: Prospective study of the natural history of infectious mononucleosis caused by Epstein-Barr virus. J Am Board Fam Pract . 2001, 14:234-42.

6. Ader F, Chatellier D, Le Berre R, Morand P, Fourrier F: Fulminant Epstein-Barr virus (EBV) hepatitis in a young immunocompetent subject. Med Mal Infect. 2006, 36:396-8.

7. Vento S, Guella L, Mirandola F, Cainelli F, Di Perri G, Solbiati M, Ferraro T, Concia E: EpsteinBarr virus as a trigger for autoimmune hepatitis in susceptible individuals. Lancet . 1995, 346:608-9.

8. Kuzman I, Kirac P, Kuzman T, Puljiz I, Bilic V: Spontaneous rupture of the spleen in infectious mononucleosis: Case report and review of the literature. Acta Med Croatica. 2003, 57:141-3.

9. Chen CM, Chao K, Su IJ: Acute primary epstein-barr virus infection presenting as acute abdomen. Pediatr Infect Dis J . 1991, 10:471-3.

10. Dahlstrom AK, Louie A, Hanna AA: Epstein barr virus (ebv) infection - A common cause of recurrent abdominal pain (rap) in children. J Ped Gastroenterology \& Nutrition . 2004, 39:379. 